\title{
Capturing Laser Induced Dynamics of Reactive Materials via Ultrafast Transmission Electron Microscopy
}

Tugba Isik ${ }^{1}$, Ceren Yilmaz Akkaya ${ }^{2}$ and Volkan Ortalan ${ }^{3}$

${ }^{1}$ School of Mechanical Engineering, Purdue University, United States, ${ }^{2}$ Department of Materials Science and Engineering, University of Connecticut, Storrs, Connecticut, United States, ${ }^{3}$ Department of Materials Science and Engineering, University of Connecticut, United States

Conventional transmission electron microscopes (TEMs) spatially resolve microstructural details of phase, structure, and morphology at the atomic scale; however, they are not capable of capturing fast dynamic events required for fundamental understanding of countless materials phenomena. Temporal resolution of the collected data is limited by camera acquisition rate, which is several orders of magnitude slower than many dynamic processes relevant to materials science. Reactive and energetic materials are known to release large amount of heat via rapid exothermic chemical reactions ${ }^{1-3}$. The response of these materials to impact and ultrasonic excitation has been heavily studied in the past years to explain the initiation and heat dissipation mechanisms ${ }^{4,5}$. It was experimentally proven that energetic materials can react below their desired threshold and hot-spot formation has been dominantly accepted as the underlying mechanism ${ }^{6}$. This theory suggests mechanical energy localization under impact due to formation of stress concentration points. As a result, temperature of the material increases locally causing initiation. Despite the considerable number of works conducted on this topic, most of the published experimental studies are limited to macrostructural level and lack the temporal resolution needed to explore initiation mechanisms of these irreversible reactions.

Ultrafast transmission electron microscopy (UTEM) has immense potential in discovering the fundamental processes leading to reaction initiation and evolution in reactive materials. While samples are excited with a nanosecond pulsed laser to trigger reactions via photothermal processes, a second pulse with a known time delay is used to stimulate photoemission of electrons from the cathode. Thanks to the generated intense electron packages, snapshot images and diffraction patterns can be collected with high temporal resolution at welldefined delay times. This single-shot approach makes it possible to capture various stages of an irreversible processes and understand how a dynamic process evolves.

In this presentation, time resolved in situ techniques in transmission electron microscopy will be demonstrated on energetic molecular crystals. These crystals were selected for their widespread applications in various fields as well as the challenges they present with high electron beam sensitivity and high reaction speed. This work aims to understand reaction mechanism of energetic materials under laser irradiation and determine transient structures that are not possible to detect with any other method. Figure 1 represents a set of experiments performed on a selected reactive molecular crystal. Bright field single-shot images of the crystals were collected before and after the reaction as well as at different time delays. Before and after structures were also imaged using electron packages generated with 10 laser pulses as a reference to single-shot image quality.

In order to estimate the temperature profile of pulsed laser heating of the samples, we utilized finite elements simulations using COMSOL Multiphysics. After appropriate modelling of the substrate, crystals and the laser parameters, time dependent temperature profile of the specimen at the start of the reaction was calculated. This information helps us to distinguish possible reaction processes that can be involved in the experiments. In this talk, examples of single-shot UTEM studies of reactive materials will be presented. Short lived transient processes involved in reactions will be analyzed in conjunction with thermal simulation results and material dynamics will be discussed to obtain new insights into the reaction processes.

\section{Acknowledgements}


This work was supported by the Young Investigator Program of Department of Defense Office of Naval Research (CBET-1437219) and the U.S. Department of Defense, Office of Naval Research, MURI Contract No. N00014-16-1-2557.

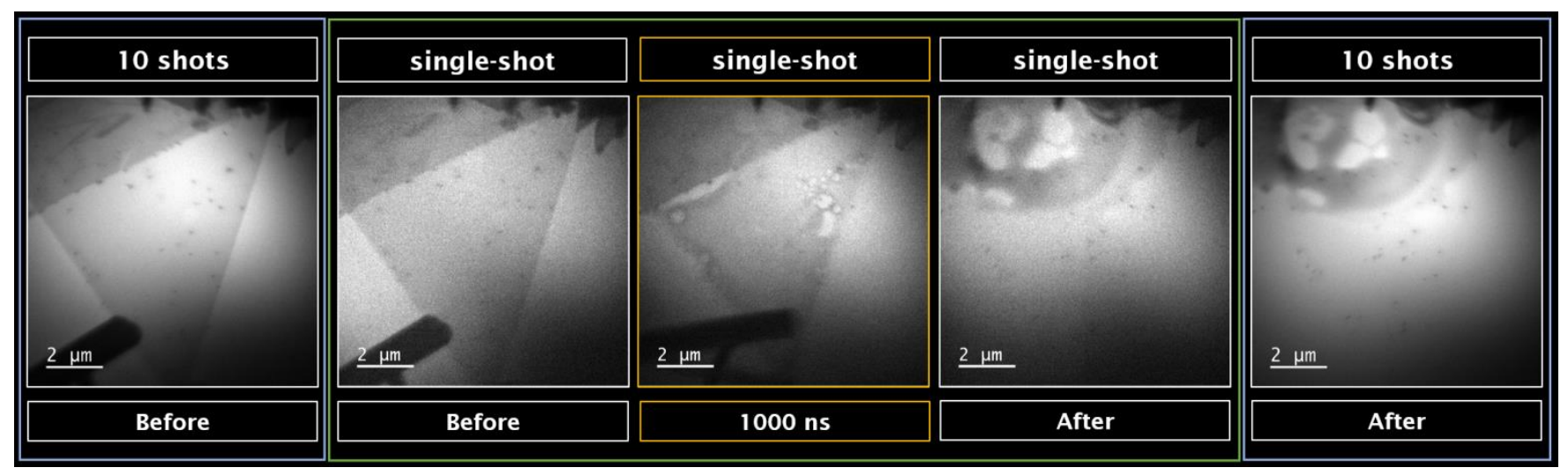

Figure 1. Figure 1: Bright field images of a selected reactive molecular crystal. The first and last images were collected with 10 subsequent pulses and added to the figure to emphasize the image quality of single-shot images. Third image is the single-shot image taken after $1000 \mathrm{~ns}$ after sample excitation showing transient state of the reaction.

\section{References}

1. Dreizin, E. L. \&Schoenitz, M. Mechanochemically prepared reactive and energetic materials: a review. J. Mater. Sci.52, 11789-11809 (2017).

2. Dreizin, E. L. Metal-based reactive nanomaterials. Prog. Energy Combust. Sci.35, 141-167 (2009).

3. Badgujar, D. M., Talawar, M. B., Asthana, S. N. \&Mahulikar, P. P. Advances in science and technology of modern energetic materials : An overview. 151, 289-305 (2008).

4. Casey, A. D. et al. Dynamic imaging of the temperature field within an energetic composite using phosphor thermography. Appl. Opt.58, 4320 (2019).

5. Mares, J. O. et al. Thermal and mechanical response of PBX 9501 under contact excitation. J. Appl. Phys.113, (2013).

6. Kerschen, N. E. et al. X-ray Phase Contrast Imaging of the Impact of a Single HMX Particle in a Polymeric Matrix. Propellants, Explos. Pyrotech.44, 447-454 (2019). 